\title{
DELIVERING NEONATAL DRUGS RESEARCH: ACCEPTABILITY OF DRIED BLOOD SPOT SAMPLING FOR PHARMACOKINETIC RESEARCH IN PREMATURE INFANTS
}

\author{
C. Soto ${ }^{1}$, H. Terry ${ }^{1}$, H. Pandya ${ }^{1}$, D.J. Field ${ }^{2}$, V. Kairamkonda ${ }^{3}$, N. Spooner ${ }^{4}$, E.M. Boyle ${ }^{2}$ \\ ${ }^{1}$ Infection, Immunity and Inflammation, ${ }^{2}$ Department of Health Sciences, University of Leicester, ${ }^{3}$ Neonatal \\ Unit, University Hospitals Leicester, Leicester, ${ }^{4}$ GlaxoSmithKline, Ware, UK
}

Background: Pharmacokinetic (PK) studies are difficult to conduct in neonates, due to the repeated need for blood sampling. A PK study of caffeine was undertaken using dried blood spots (DBS) taken opportunistically during other blood tests. We designed a qualitative study to investigate the acceptability of this novel technique to parents and nurses on the neonatal unit.

Objective: To determine the acceptability of using DBS for pharmacokinetic research in premature infants.

Design/methods: Nineteen parents, and thirty-one nurses were recruited during the pharmacokinetic study and participated in semi-structured interviews. Interviews were analysed through the constant comparative method.

Results: Parents and nurses felt that research is important to improve outcomes for future neonates, although this was balanced by a need to protect the baby from undue harm or distress. The clinical condition of each baby was deemed important in deciding appropriate sampling volumes and times. Both groups expressed concern that larger sampling volumes would put the baby under additional stress, and lead to more blood transfusions. Opportunistic sampling was viewed positively by parents and nurses, as it avoided further pain to the baby and supported minimal handling. Both parents and nurses felt that using minimal blood volumes and opportunistic sampling made it easier for parents to consent, and thus facilitated successful recruitment.

Conclusions: Parents and nurses found DBS sampling an acceptable method for PK trials in premature neonates, especially when samples are taken opportunistically. DBS enables drug monitoring in vulnerable neonates and the safer development and administration of drug therapies. 